\title{
Prediction of Ferromagnetic Ground State of NaCl-type FeN
}

\author{
A. Filippetti and W. E. Pickett \\ Department of Physics, University of California - Davis, Davis, Ca 95616
}

\begin{abstract}
$\mathrm{Ab}$ initio results for structural and electronic properties of $\mathrm{NaCl}$-type $\mathrm{FeN}$ are presented. Calculations are performed within a plane-wave and ultrasoft pseudopotential framework. Competition among paramagnetic, ferromagnetic and some possible antiferromagnetic phases is examined. We find the ferromagnetic phase stable overall as observed. Stabilization over the unpolarized phase is obtained by splitting one flat $t_{2 g}$-type band crossing the Fermi energy. Comparison with $\mathrm{CrN}$ reveals that the magnetic ordering of $\mathrm{CrN}$, which consists of double-ferromagnetic sheets compensating along the [110] direction of the cubic cell and stabilized by the distortion of the squared symmetry on (100) plane, is not effective for FeN. The different behavior of $\mathrm{FeN}$ and $\mathrm{CrN}$ can be traced back to the smaller ionicity and magnetization of the former.
\end{abstract}

71., 75., 71.15.Hx, 75.50.Ee

\section{INTRODUCTION}

Stable compound 1 formed by Fe and N, such as $\gamma^{\prime}$ $\mathrm{Fe}_{4} \mathrm{~N}$ and $\zeta-\mathrm{Fe}_{2} \mathrm{~N}$, or quasi-stable like $\alpha^{\prime \prime}-\mathrm{Fe}_{16} \mathrm{~N}$ have been known for some time. On the other hand, almost nothing was reported about FeN until the work of Heiman et al.3, where for the first time, they synthesized $\mathrm{Fe}_{x} \mathrm{~N}_{1-x}$ with $0.4<x<0.75$. In this work FeN was found to be ferromagnetic. Successive studies found FeN in $\mathrm{NaCl}$-type crystal structure 4 , and non-ferromagnetic at room temperature 5 . More recent works are in contradiction with the earlier ones: Morita et al. 6 reported FeN antiferromagnet at temperatures below $100 \mathrm{~K}$. Moreover, Suzuki et al. determined the FeN structure to be of $\mathrm{ZnS}$ fcc type. They also found their sample to exhibit a mictomagnetic character that was suggested be related to the antiferromagnetism of FeN.

Regarding the structure, it seems clear that FeN can assume both zincblende and rocksalt structure, depending on the sample history, and both of them may contain some deficiencies of $\mathrm{N}$ atoms. Regarding magnetic properties, recent Mössbauer spectroscopy measurements suggested that $\mathrm{NaCl}$-type $\mathrm{FeN}$ is antiferromagnetic 8 , with Néel temperature around room temperature. However, Mössbauer spectroscopy is not able to determine completely magnetic ordering.

For both structural and magnetic properties ab initio calculations are suited to furnish a prediction about the ground state of FeN, and to help guide understanding of experimental results. Here we investigate the relative stability of paramagnetic (PM), ferromagnetic (FM) and some possible antiferromagnetic (AFM) phases of NaCl-type FeN that have been observed or suggested for other transition-metal nitride (or oxide or carbide) compounds. Compared to other transition-metal nitrides (TMNs), it has been noticed 11 that NaCl-type FeN shows electronic properties close to those of $\mathrm{CrN}$ that we recently investigated 10, e.g. the Fermi energy falls exactly on a pronounced peak of the density of states, mainly formed by $\mathrm{Fe} d$-like flat bands. In Ref. 11 the authors come to the conclusion, on the basis of the rigid band model to evaluate the DOS and the Stoner condition of ferromagnetism, that only $\mathrm{CrN}, \mathrm{FeN}$ and $\mathrm{CoN}$ in rocksalt phase are likely to be stabilized by magnetic ordering.

Comparison with $\mathrm{CrN}$ is particularly interesting because it undergoes a cubic-to-orthorhombic transition accompanying the PM to AFM ordering at a Néel temperature close to the room temperature, with AFM phase formed by double ferromagnetic sheets compensating along the [110] direction. The structural distortion is decisive in determining the most stable among the different AFM phases, and stress relief is likely to be the ultimate driving force towards distortion. If such an involved mechanism of magnetic-ordering is driven by the high DOS at Fermi level, it could be argued that a similar magnetic ordering occurs for FeN as well. Thus, for possible AFM phases, we decide to consider here the [110]double and single sheet AFM arrangements (indicated as $\mathrm{AFM}_{[110}^{2}$ and $\mathrm{AFM}_{[110]}^{1}$, respectively and described fully in Ref.10), as well as the $\mathrm{AFM}_{[111]}$ phase (very common for NaCl-type transition metal oxides), consisting of single ferromagnetic sheets alternating along the [111] direction.

Equilibrium lattice constants, energies, and magnetic moments are presented for all the considered phases. Also, band structures and density of states (DOS's) are presented for PM and FM phases. Our local-spin-density calculations are performed in a plane-wave and ultrasoft pseudopotential framework 12 . Use of ultrasoft pseudopotentials allow us to obtain well converged results for a cutoff energy equal to 30 Ryd. We used sets of 10 to 110 special k-points (depending on the structure) for the selfconsistent calculations, up to $280 \mathrm{k}$-points to evaluate the DOS.

\section{STRUCTURE}

Experimentally it was found for the lattice constant of FeN in rocksalt and zincblende phase $a_{0}^{r s}=8.5$ bohr 8 and $a_{0}^{z b}=8.1-8.2$ bohr 8 , respectively. This result is somewhat surprising; indeed, assuming the same anion-cation bond length in the two phases, the relation between lattice constants would be $a_{0}^{r s}=(\sqrt{3} / 2) a_{0}^{z b}<a_{0}^{z b}$. Al- 
though this crude approximation considerably overestimates the actual difference between the two structures, it is generally true that in most cases the rocksalt phase have larger volume. Doubts about the measurements are confirmed by a recent theoretical work11 where structural properties for transition metal nitrides are calculated. They found for $a_{0}^{z b}$ a value in good agreement with experiments, but a much smaller $a_{0}^{r s} \sim 7.5$ bohr in the paramagnetic phase. The discrepancy with experiment is so large that it seems unlikely it could be due to different magnetic orderings. Also, in Ref. 11 the equilibrium lattice constant for other phases (ferromagnetic and a couple of antiferromagnetic phases) was evaluated, but no relevant changes on $a_{0}^{r s}$ were found. Finally, the value $a_{0}^{r s} \sim 7.5 \mathrm{bohr}$ is consistent with that of other rocksalt TMN structures. For instance, experiments assign to AFM CrN a value $a_{0}^{r s} \sim 7.8$ bohr, and we should expect for FeN a somewhat smaller lattice constant.

To try to explain the disagreement with experiments we consider here other AFM phases. In particular, among the AFM phases examined in Ref. 11, the $\mathrm{AFM}_{[110]}$ was not taken into account. The PM to $\mathrm{AFM}_{[110]}^{2}$ transition is found to produce, for $\mathrm{CrN}$, a large increase of lattice constant $10(\sim 2 \%)$, although not large enough to account for the discrepancy with experiments of $\mathrm{FeN}$, and the largest energy gain with respect to the PM phase. In Table If we report our calculated values of $a_{0}^{r s}$ for the corresponding magnetic orderings. Evidently, contrary to what happens for $\mathrm{CrN}$, the structure of FeN is basically unaffected by magnetic ordering, and only a $0.4 \%$ increase of $a_{0}^{r s}$ is found in the FM phase with respect to the PM. Our results are in substantial agreement with Ref. 11 (they found $a_{0}=7.47$ and 7.54 for PM and AFM phases, respectively).

From our calculations, it results that NaCl-type FeN is ferromagnetic. The energy gain of FM phase with respect to the PM is less than $0.1 \mathrm{eV}$ per formula unit, i.e. significantly smaller than in case of CrN. Also, the competition between FM and the examined AFM phases is very close (energy differences are few hundredths of $\mathrm{eV})$. To be confident about the precision of our calculations, we evaluated the FM-AFM energy differences by performing FM calculations in the same symmetry of all the AFM structures considered, and tested the differences for sets of special K-points of increasing size. We conclude that, among the AFM phases, the lowest in energy is $\mathrm{AFM}_{[110]}^{1}$, also the lowest among the cubic phases

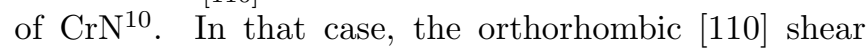
distortion of $\mathrm{CrN}$ produces a further energy gain for the $\mathrm{AFM}_{[110]}^{2}$ structure (anisotropic in the (100) plane due to magnetic ordering) that causes the latter to be the most stable phase overall. To investigate the effect of planar distortion on FeN we applied the same distortion experimentally observed for $\mathrm{CrN}$ (i.e. a $\sim 2 \%$ reduction of the bisection angle at the squared base of the tetragonal cell. Surprisingly, we find very little change in the energy with respect to the cubic $\mathrm{AFM}_{[110]}^{2}$ phase (the increase is just $\sim 2 \mathrm{meV})$

Thus, notwithstanding the similarity of DOS at Fermi level, $\mathrm{CrN}$ and FeN are stabilized by very different magnetic order and structure. In Table f we report the magnetization $m$ (per formula unit, i.e. per couple Fe-N) for the considered phases of FeN. The FM phase has the highest magnetization $\left(1.65 \mu_{B}\right)$, of which $\sim 0.15 \mu_{B}$ comes from polarization of $\mathrm{N}$, and $\sim 1.5 \mu_{B}$ from polarization of $\mathrm{Fe}$. For $\mathrm{CrN}$ the magnetization is more that 2 $\mu_{B}$ per formula unit. Also, the charge transfer is different. For FeN it is equal to about one electron, so that the approximate ionic configuration $\mathrm{Fe}^{+1} \mathrm{~N}^{-1}$ can be deduced, i.e. Fe is near a $d^{7}$ configuration. In case of $\mathrm{CrN}$, the charge transfer is larger (close to 2 electrons), due to the higher electronegativity of $\mathrm{Fe}$ with respect to $\mathrm{Cr}$. i.e. to the smaller electronegativity difference with $\mathrm{N}$. The higher covalency of FeN contributes to it having a smaller lattice constant, whereas $\mathrm{CrN}$ has larger ionicity and polarization.

In Ref. 10 it is argued that for $\mathrm{CrN}$ the energy gain from the planar distortion is due to a relief of tensile stress stored in the bonds between metal atoms with antiparallel spins. This stress can be conveniently defined as the excess stress produced by a transition from the $\mathrm{AFM}_{[110]}^{1}$ phase (where there is no stress by symmetry) and the $\mathrm{AFM}_{[110]}^{2}$ cubic phase with the same lattice constant. The transition produces a planar anisotropic stress, whose component $\tau_{[1 \overline{1} 0]}$ is tensile, i.e. the spinantiparallel metal atoms tend to get closer each other. In light of our results for FeN, we speculate that this magnetic order-derived stress is sensitive to the magnitude of magnetization. Of course, the different bond length (slightly larger for $\mathrm{CrN}$ than for FeN) plays a role as well and could justify in itself a different behavior.

\section{ELECTRONIC PROPERTIES}

In Figure 1 the DOS for the PM phase of FeN is shown. In this energy region, the total DOS (top panel) is almost completely made up by $\mathrm{d}$ states of Fe and $\mathrm{p}$ states of $\mathrm{N}$ (bottom panel). The d-like DOS shows two high peaks close to the Fermi energy $\left(E_{F}\right)$. The lowest in energy is completely occupied and is composed by a mixture of $75 \% t_{2 g}$-type and $25 \% e_{g}$-type character It is this peak which falls at $\mathrm{E}_{F}$ in paramagnetic $\mathrm{CrN} 10$ (a rigid band model works well for the PM phase of this system). The second peak in the d DOS is entirely $t_{2 g}$ in character, and is centered at $\mathrm{E}_{F}$. The peaks located between -8 $\mathrm{eV}$ and $-4 \mathrm{eV}$ are mostly $\mathrm{N} \mathrm{p}$, with a mixture of $t_{2 g^{-}}$ type and (predominantly) $e_{g}$-type states. The $\mathrm{N}$ p-like DOS gives an appreciable contribution to the DOS at the Fermi energy as well, unlike $\mathrm{CrN}$ for which only d-type states contribute. This DOS peak at $\mathrm{E}_{F}$ arises from a strong $d p \sigma$ antibonding band.

The effect of FM ordering is easily visible in Figure 2. Now the Fe d-like DOS (middle panel) is shown de- 
composed in $t_{2 g}$ and $e_{g}$ states. The two d-type peaks are now split by an exchange splitting of roughly $1.6 \mathrm{eV}$. After this splitting both peaks in the spin up channels are completely occupied, whereas the upper peak of the spin down channel is empty. Thus, 3 electrons per $\mathrm{Fe}$ atom saturate almost completely three $t_{2 g}^{\uparrow}$ states, and $\sim$ 1.5 electron charge of mostly $t_{2 g}$ type remains unpaired. Consistently with a cation to anion charge transfer of $\sim$ 1 electron, i.e. with a $d^{7}$ configuration for $\mathrm{Fe}$, there are 2.5 electrons occuping nearly paired $e_{g}$ states. The $\mathrm{N} \mathrm{p}$ character in the DOS contributes to the magnetization, reflecting the $\mathrm{N}$ moment of $\sim 0.15 \mu_{B}$.

The same features can be seen in the band structure. In Figure 3 the PM FeN band structure is presented. It is easy to distinguish the very flat $t_{2 g}$-type band crossing the Fermi level through $\Delta, Z$ and $Q$ directions, that produces the half-occupied peak of PM DOS shown in Figure 1. After FM ordering (Figure 4), the band is split, in such a way that the up band (left panel) is shifted $\sim 1.5-2 \mathrm{eV}$ down to the Fermi energy, and the down band rises up slightly, but still remains partially occupied. Evidently, much of the minority DOS peak at $\sim 0.8 \mathrm{eV}$ arises from regions away from the symmetry lines plotted in Figure 4.

A feature observable in Figure 4 is that the spin splitting for some bands is strongly $\mathrm{k}$-dependent, unlike the Stoner picture in which the splitting is rigid. For example, all bands at $\Gamma$ are clustered within $1 \mathrm{eV}$ in the paramagnetic case and remain so in the minority bands, whereas in the majority bands they span a range of nearly $2 \mathrm{eV}$. Such non-rigid behavior has been observed in other transition metal compounds, and reflects a combination of (i) an anisotropic exchange potential (around the Fe atom) and (ii) hybridization between d orbitals on a strongly magnetic atom (Fe) and p orbitals on a weakly magnetic atom $(\mathrm{N})$.

\section{CONCLUSIONS}

In this paper the structure of NaCl-type FeN has been investigated by ab initio calculations. The ferromagnetic phase is found to be stable against the paramagnetic and all the antiferromagnetic phases here considered. However, the energy differences between the magnetic phases are within few hundredths of $\mathrm{eV}$, and this fact, coupled with sensitivity of the magnetism to stoichiometry, could explain the experimental variations reported in the literature. In order to get insights into the magnetic ordering mechanisms, a comparison with $\mathrm{CrN}$ was made. Although for both compounds a sharp $d$-like peak is present at $\mathrm{E}_{F}$, the differences in charge transfer, magnetization and differing wavefunction character at $\mathrm{E}_{F}$ lead to very different magnetic properties. In particular, the orthorhombic distortion that is able to stabilize AFM CrN has no effect on FeN. Since the distortion indicates the presence of tensile stress stored on bonds between metal atoms with antiparallel spins, we suspect that the stress could depend sensitively on the magnitude of magnetization.

The relief of stress is now accepted to be an important factor in the structure and properties of surfaces and interfaces. Our results strongly suggest that the stress relief may be instrumental in determining the magnetic order in compounds such as FeN.

\section{ACKNOWLEDGMENTS}

This work was supported by National Science Foundation Grant No. DMR-9802076. Computations were carried out at the San Diego Supercomputing Center and at the Maui High Performance Computing Center.

${ }^{1}$ M. Hansen, Constitution of Binary Alloys (McGraw-Hill, New York, 1958), p.670.

${ }^{2}$ T. K. Kim and M. Takahashi, Appl. Phys. Lett. 20, 492 (1972).

${ }^{3}$ N. Heiman and N. S. Kazama, J. Appl. Phys. 52, 3562 (1981).

${ }^{4}$ A. Oueldennaoua, E. Bauer-Grosse, M. Foos, and C. Frantz, Scr. Metall. 19, 1503 (1985).

${ }^{5}$ K. Suzuki, H. Yoshida, and H. Fujimori, Proc. Int. Symp. on Physics of Magnetic Materials, Sendai (World Scientific, Singapore, 1987), p.93.

${ }^{6}$ H. Morita, H. Yoshida, T. Kaneko, H. Fujimori and K. Suzuki, Proc. 2nd Int. Symp. on Physics of Magnetic Materials, Beijing (International Academic Publishers, Beijing, 1992), p.633.

${ }^{7}$ K. Suzuki, H. Morita, T. Kaneko, H. Yoshida and H. Fujimori, J. Alloys Comp. 201, 11 (1993).

${ }^{8}$ H. Nakagawa, S. Nasu, M. Takahashi and F. Kanamaru, Hyperfine Interact. 69, 445 (1991).

${ }^{9}$ T. Hinomura and S. Nasu, Physica B 237-238 557 (1997).

${ }^{10}$ A. Filippetti, W. E. Pickett, and B. M. Klein, condmat/9808266.

${ }^{11}$ H. Shimizu, M. Shirai and N. Suzuki, J. Phys. Soc. Jpn. 66, 3147 (1997); 67, 922 (1998).

12 D. Vanderbilt, Phys. Rev. B 32, 8412 (1985); K. Laasonen, A. Pasquarello, R. Car, C. Lee, and D. Vanderbilt, Phys. Rev. B 47, 10142 (1993). 
TABLE I. Equilibrium lattice constant $a_{0}$ (in bohr), energy $E$ (in $\mathrm{eV} /$ formula unit) and magnetic moment $m$ (in $\mu_{B} /$ metal atom) of NaCl-type FeN calculated for different magnetic phases. Energies are referred to the lowest one, i.e. that of FM phase.

\begin{tabular}{cccccc}
\hline \hline & $\mathrm{FM}$ & $\mathrm{PM}$ & $\mathrm{AFM}_{[110]}^{1}$ & $\mathrm{AFM}_{[110]}^{2}$ & $\mathrm{AFM}_{[111]}$ \\
\hline$a_{0}$ & 7.55 & 7.52 & 7.55 & 7.52 & 7.52 \\
$E$ & 0 & 0.093 & 0.017 & 0.042 & 0.028 \\
\hline$m$ & 1.65 & & 1.44 & 1.46 & 1.15 \\
\hline \hline
\end{tabular}

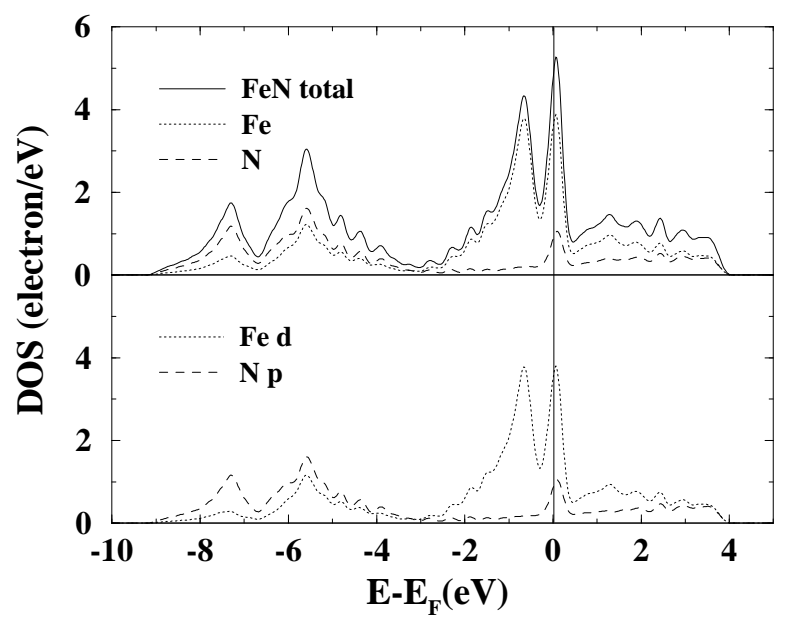

FIG. 1. Top panel: total and atom by atom decomposed DOS of PM NaCl-type FeN. Bottom panel: Fe $d$-type and N $p$-type contributions to the total DOS. In the shown energy window this two angular components are largely dominant.

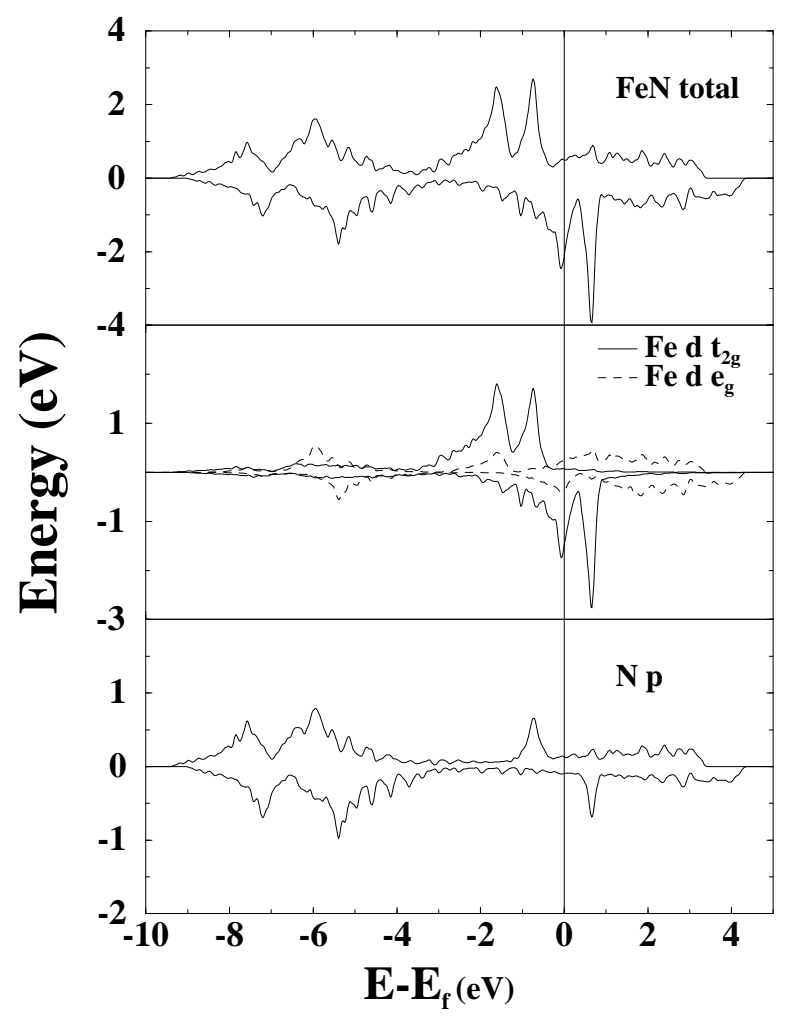

FIG. 2. Top panel: total DOS of FM NaCl-type FeN for spin up and down charge. Middle: Fe $d t_{2 g}$-type (solid line) and $e_{g}$-type (dashed) contributions to DOS. Bottom: $\mathrm{N} p$-type contribution. 


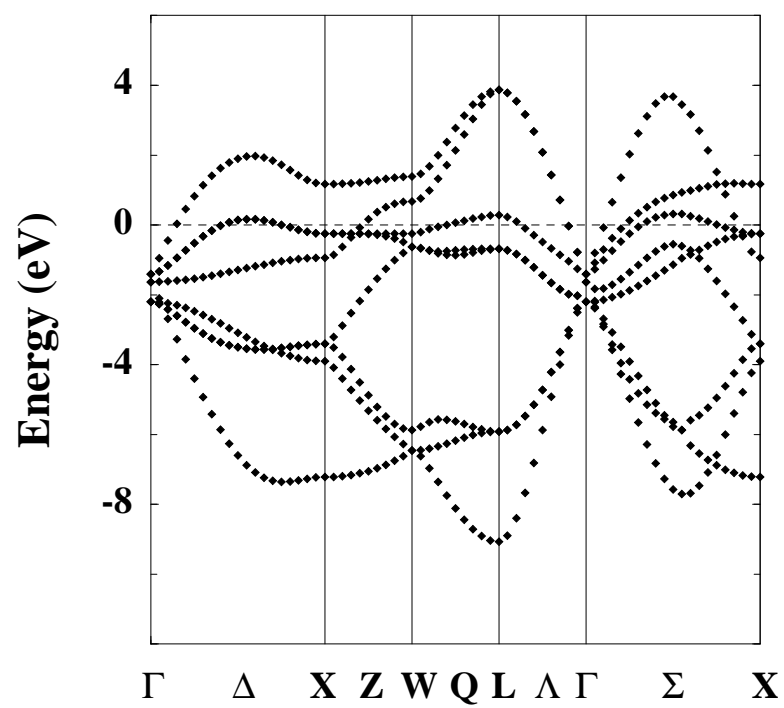

FIG. 3. Band structure for PM NaCl-type FeN. A flat band of $t_{2 g}$ character running from $\Gamma$ to $L$ almost in touch with the Fermi level is responsible for the pronounced peak of DOS at the Fermi Energy.
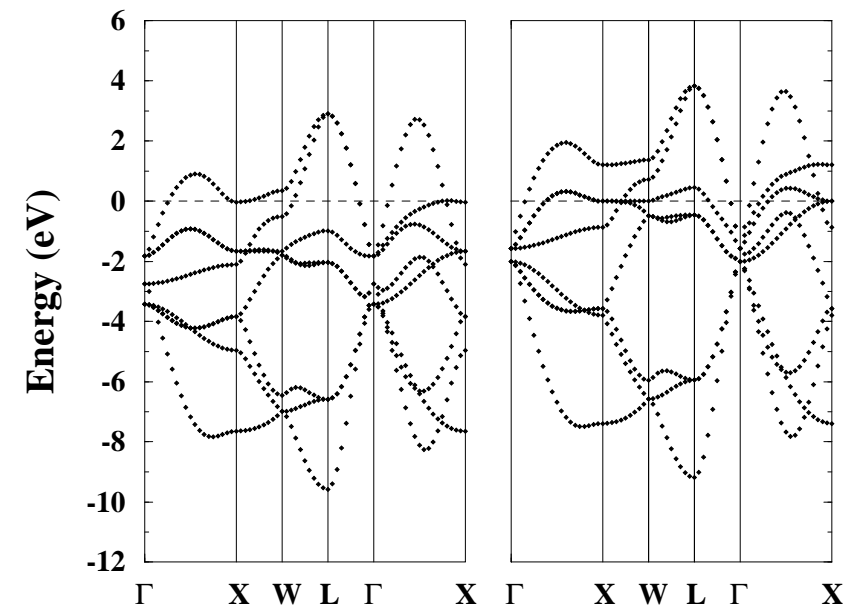

FIG. 4. Band structure for FM NaCl-type FeN. Left and right panels refer to spin up and down bands, respectively. Directions and K-points are the same of the previous Figure. 